\title{
A Combined Estimator of Regression Models with Measurement Errors
}

\author{
Bai Huang*, Tae-Hwy Lee ${ }^{\dagger}$, Aman Ullah \\ June 2017
}

\begin{abstract}
When the regressors are observed with measurement errors, the OLS estimator is inconsistent and typically the use of the IV estimator is recommended. In this paper we place this recommendation under scrutiny, especially (i) when the instruments are weak and (ii) when there are many instruments. Following Hansen (2017), we use the Hausman (1978) test for errors in variables to combine OLS and IV estimators. The combined estimator has the asymptotic risk strictly less than that of the IV estimator. Then we show some useful findings for small samples based on the Monte Carlo simulations. In terms of the mean squared error risk, we find that (a) typically OLS gets worse as the measurement error gets larger while IV is more robust and better than OLS, (b) OLS can be better than IV when the measurement error is small, and (c) the combined estimator outperforms IV as the asymptotic result predicts. (a) and (b) are true only when the instruments are not weak and when there are not many instruments. However, when the instruments are weak or when there are many instruments: (c) still holds as it is a theorem, but (a), (b) turn out to become quite the opposite, i.e., OLS can be much better than IV even when measurement error is large. This happens because IV is known to be inconsistent with weak instruments and many instruments (Staiger and Stock 1997, Bekker 1994), and can be much worse than OLS, making the combined estimator close to OLS. In that case, the typical recommendation to use IV should be guided by the combined estimator, and IV and the combined estimator need to be regularized for weak instruments and many instruments.
\end{abstract}

Key Words: OLS, IV, measurement errors, weak instruments, many instruments, regularized combined estimator.

JEL Classification: C13, C33, C52

${ }^{*}$ Department of Economics, University of California, Riverside, CA 92521. bai.huang@email.ucr.edu

${ }^{\dagger}$ Department of Economics, University of California, Riverside, CA 92521. tae.lee@ucr.edu

${ }^{\ddagger}$ Department of Economics, University of California, Riverside, CA 92521. aman.ullah@ucr.edu 


\section{Introduction}

When some of the regressors have errors in variables, the ordinary least squares (OLS) estimator is inconsistent and typically the instrumental variables (IV) estimator is recommended. In this paper we look into this recommendation closely and propose an estimator providing improvement over the IV as well as OLS estimators in terms of the total mean squared error risk. Motivated by Hansen (2017), we use the Hausman (1978) test for errors in variable to combine OLS and IV. Further, following Hansen (2017), the asymptotic distribution and the asymptotic risk of the combined estimator using a local asymptotic framework are presented. This shows that, if the regressor dimension exceeds two, the asymptotic risk of the combined estimator is strictly less than that of the IV estimator.

Our simulation result shows that the combined estimator can substantially reduce finite sample risk relatively to the IV estimator, as well as relative to the OLS estimator for

moderate degrees of measurement errors. In terms of the mean squared error risk, we find that (a) typically OLS gets worse as the measurement error gets larger while IV is more robust and better than OLS, (b) OLS can be better than IV when the measurement error is small, and (c) the combined estimator outperforms IV as the asymptotic result predicts. (a) and (b) are true only when the instruments are not weak and when there are not many instruments.

However, when the instruments are weak or when there are many instruments, (c) still holds as it is a theorem, but (a), (b) turn out to become quite the opposite, i.e., OLS can be much better than IV even when measurement error is large. This happens because IV is known to be inconsistent with weak instruments and many instruments (Staiger and Stock 1997, Bekker 1994), and can be much worse than OLS, making the combined estimator become closer to OLS. In that case, the typical recommendation to use IV should be guided by the combined estimator and we need to regularize the OLS, IV, and the combined estimators 
to reduce the number of instruments and to shrink the effect of the weak instruments. The findings from this paper suggest that in the presence of many instruments and weak instruments, the regularization would be the first step to recover the consistency of IV before using it to construct a regularized combined estimator. Weak instruments and many instruments need to be treated before constructing the combined estimator.

The rest of the paper is organized as follows. Section 2 reviews issues in the regression model with measurement errors. Section 3 presents the combined estimator and summarizes its asymptotic distribution and the asymptotic risk. Monte Carlo simulation is provided in Section 4. Concluding remarks follows in Section 5.

\section{Regression Model with Measurement Errors}

We consider the linear regression

$$
y_{i}=x_{i}^{* \prime} \beta+\varepsilon_{1 i}
$$

where $y_{i}$ and $\varepsilon_{1 i}$ are scalars, $x_{i}^{*}$ is a $q \times 1$ vector, and $\sigma_{\varepsilon_{1}}^{2}=E\left(\varepsilon_{1 i}^{2}\right)$. The covariates $x_{i}^{*}$ is unobserved but measured with measurement error $\varepsilon_{2 i}$ such that

$$
x_{i}=x_{i}^{*}+\varepsilon_{2 i}
$$

is observed and $\Sigma_{\varepsilon_{2}}=E\left(\varepsilon_{2 i} \varepsilon_{2 i}^{\prime}\right)$. Our goal is to estimate $\beta$. Let $z_{i}$ be an $\ell \times 1(\ell \geq q)$ vector of instruments that are related to $x_{i}^{*}$

$$
x_{i}^{*}=\Pi z_{i}+\varepsilon_{3 i}
$$

where $x_{i}^{*}, \varepsilon_{2 i}, \varepsilon_{3 i}$ are $q \times 1$ vectors. For simplicity, we assume that all of $\varepsilon_{1 i}, \varepsilon_{2 i}, \varepsilon_{3 i}$ are uncorrelated each other, and we also assume that $\Sigma_{\varepsilon_{2}}=\operatorname{diag}\left(\sigma_{1}^{2}, \sigma_{2}^{2}, \cdots, \sigma_{q}^{2}\right)$ is diagonal.

Then the model is written as

$$
y_{i}=x_{i}^{* \prime} \beta+\varepsilon_{1 i}=x_{i}^{\prime} \beta+\left(\varepsilon_{1 i}-\varepsilon_{2 i}^{\prime} \beta\right) \equiv x_{i}^{\prime} \beta+u_{i}
$$


where $u_{i}=\varepsilon_{1 i}-\varepsilon_{2 i}^{\prime} \beta$ and $\sigma_{u}^{2}=E\left(u_{i}^{2}\right)=\sigma_{\varepsilon_{1}}^{2}+\beta^{\prime} \Sigma_{\varepsilon_{2}} \beta$. Also,

$$
x_{i}=x_{i}^{*}+\varepsilon_{2 i}=\Pi z_{i}+\varepsilon_{3 i}+\varepsilon_{2 i} \equiv \Pi z_{i}+v_{i},
$$

where $v_{i}=\left(\varepsilon_{3 i}+\varepsilon_{2 i}\right)$ and $\Sigma_{v}=E\left(v_{i} v_{i}^{\prime}\right)=\Sigma_{\varepsilon_{2}}+\Sigma_{\varepsilon_{3}}$.

The OLS estimator

$$
\hat{\beta}_{0}=\left(X^{\prime} X\right)^{-1}\left(X^{\prime} y\right)
$$

may be inconsistent because

$$
\begin{aligned}
\hat{\beta}_{0}-\beta \stackrel{p}{\rightarrow}\left[E\left(x_{i} x_{i}^{\prime}\right)\right]^{-1}\left(E x_{i} u_{i}\right) & =\left[E\left(x_{i} x_{i}^{\prime}\right)\right]^{-1}\left[-E\left(\varepsilon_{2 i} \varepsilon_{2 i}^{\prime}\right) \beta\right] \\
& =\left[E\left(x_{i} x_{i}^{\prime}\right)\right]^{-1}\left[-\Sigma_{\varepsilon_{2}} \beta\right] \\
& =\left[E\left(x_{i} x_{i}^{\prime}\right)\right]^{-1}\left[-\sigma_{\varepsilon_{2}}^{2} \beta\right] .
\end{aligned}
$$

The last line uses the assumption that the measurement errors to all regressors have the same degree (for simplicity), i.e.,

$$
\Sigma_{\varepsilon_{2}}=\operatorname{diag}\left(\sigma_{1}^{2}, \sigma_{2}^{2}, \cdots, \sigma_{q}^{2}\right)=\sigma_{\varepsilon_{2}}^{2} I_{q}
$$

When $\sigma_{\varepsilon_{2}}^{2}=0$, the OLS estimator $\hat{\beta}_{0}$ is consistent and efficient. When $\sigma_{\varepsilon_{2}}^{2} \neq 0$, the OLS estimator $\hat{\beta}_{0}$ is inconsistent and a consistent estimator $\hat{\beta}_{1}$ using the instrument $z$ can be obtained from

$$
\hat{\beta}_{1}=\left(\hat{X}^{\prime} \hat{X}\right)^{-1}\left(\hat{X}^{\prime} y\right)
$$

where $P_{Z}=Z\left(Z^{\prime} Z\right)^{-1} Z^{\prime}$ and $\hat{X}=P_{Z} X$.

The Hausman test for the null hypothesis that $x_{i}$ has no measurement error against the alternative hypothesis that $x_{i}$ has measurement errors is to compare the two estimators $\hat{\beta}_{0}$ and $\hat{\beta}_{1}$. Then the Hausman statistic is

$$
H_{n}=n\left(\hat{\beta}_{1}-\hat{\beta}_{0}\right)^{\prime}\left(\hat{V}_{1}-\hat{V}_{0}\right)^{-1}\left(\hat{\beta}_{1}-\hat{\beta}_{0}\right)
$$


where $\hat{V}_{0}$ and $\hat{V}_{1}$ are the consistent estimators for $V_{0}=\sigma_{u}^{2}\left[E\left(x_{i} x_{i}^{\prime}\right)\right]^{-1}$ and $V_{1}=\sigma_{u}^{2}\left[E\left(x_{i} z_{i}^{\prime}\right)\left[E\left(z_{i} z_{i}^{\prime}\right)\right]^{-1} E\left(z_{i} x_{i}^{\prime}\right)\right]^{-1}$ respectively. $V_{0}$ and $V_{1}$ are the asymptotic variances of $\hat{\beta}_{0}$ and $\hat{\beta}_{1}$. If $H_{n}$ is larger than a certain critical value, $\hat{\beta}_{1}$ is considered to be the preferred estimator.

\section{A Combined Estimator}

When either $\sigma_{\varepsilon_{2}}^{2}=0$ or $\sigma_{\varepsilon_{2}}^{2} \neq 0$, it is easy to select one of $\hat{\beta}_{0}$ and $\hat{\beta}_{1}$ from the Hausman statistic for measurement errors. But when $\sigma_{\varepsilon_{2}}^{2}$ is local to zero, it may not be clear which estimator to choose. In such a case a combined estimator of the following form is considered

$$
\hat{\beta}_{c}=w \hat{\beta}_{0}+(1-w) \hat{\beta}_{1}
$$

where $w=\min \left(\frac{\tau}{H_{n}}, 1\right)$ and $\tau$ is a shrinkage parameter determined as suggested by James and Stein (1961). The asymptotic behavior of the three estimators $\hat{\beta}_{0}, \hat{\beta}_{1}, \hat{\beta}_{c}$ has been elegantly derived in Hansen (2017) for the structural econometric model. As the same asymptotic theory of Hansen (2017) applies to the present cases with measurement errors in the regressors, as summarized below. The main goal of our paper is to examine the finite sample behavior to see how the asymptotic theory carries over to the finite sample cases. Not surprisingly, the finite sample behavior of the three estimators are quite similar in Hansen (2017) and in measurement error models. Interestingly though, we find that when $q$ is large relative to the sample size $n$, the finite sample risks of $\hat{\beta}_{0}, \hat{\beta}_{1}, \hat{\beta}_{c}$ are quite different than that when $q$ is reasonably small.

The variable $x_{i}$ is exogenous if there is no measurement error, i.e., $\sigma_{\varepsilon_{2}}^{2}$ is zero. Consider the case when $\sigma_{\varepsilon_{2}}^{2}$ is local to zero

$$
\sigma_{\varepsilon_{2}}^{2}=\frac{1}{\sqrt{n}} \delta_{1}
$$

where $\delta_{1}$ is the $1 \times 1$ localizing parameter, which is the degree of measurement error. Suppose the two error terms in (4) and (5) are linearly related and write the structural equation error 
$u_{i}$ as a linear function of the reduced form equation error $v_{i}$ and the orthogonal error $\eta_{i}$

$$
\begin{aligned}
u_{i} & =\rho^{\prime} v_{i}+\eta_{i} \\
E\left(v_{i} \eta_{i}\right) & =0 .
\end{aligned}
$$

Hansen (2017) uses the local to zero exogeneity in the sense of

$$
\rho=\frac{1}{\sqrt{n}} \delta_{2}
$$

where $\delta_{2}$ is the $q \times 1$ localizing parameter. To relate $\delta_{1}$ and $\delta_{2}$, recall $u_{i}=\varepsilon_{1 i}-\varepsilon_{2 i}^{\prime} \beta$ and $v_{i}=\varepsilon_{3 i}+\varepsilon_{2 i}$, and thus

$$
\rho=\left[E\left(v_{i} v_{i}^{\prime}\right)\right]^{-1} E\left(v_{i} u_{i}\right)=\Sigma_{v}^{-1}\left(-\sigma_{\varepsilon_{2}}^{2} \beta\right)
$$

Therefore

$$
\delta_{2}=\Sigma_{v}^{-1}\left(-\delta_{1} \beta\right)
$$

or

$$
\Sigma_{v} \delta_{2}=-\delta_{1} \beta
$$

and thus the local to zero measurement error $\delta_{1}$ is a linear function of the local to zero endogeneity $\delta_{2}$. We state the following two theorems both in $\delta_{1}$ (local degree of measurement error) and $\delta_{2}$ (local degree of correlation between the structural error $u_{i}$ and the reduced form error $v_{i}$ ).

Theorem 1 (Hansen 2017). Under the conditions that conventional central limit theory applies and that the error is conditionally homoskedastic

$$
\sqrt{n}\left(\begin{array}{c}
\hat{\beta}_{0}-\beta \\
\hat{\beta}_{1}-\beta
\end{array}\right) \stackrel{d}{\rightarrow} h+\xi
$$

where

$$
h=\left(\begin{array}{c}
\sigma_{u}^{-2} V_{0} \Sigma_{v} \delta_{2} \\
0
\end{array}\right)=\left(\begin{array}{c}
-\sigma_{u}^{-2} V_{0} \beta \delta_{1} \\
0
\end{array}\right)
$$




$$
\xi \sim N(0, V)
$$

with

$$
\begin{aligned}
V & =\left(\begin{array}{ll}
V_{0} & V_{0} \\
V_{0} & V_{1}
\end{array}\right) \\
V_{0} & =\sigma_{u}^{2}\left[E\left(x_{i} x_{i}^{\prime}\right)\right]^{-1} \\
V_{1} & =\sigma_{u}^{2}\left[E\left(x_{i} z_{i}^{\prime}\right)\left[E\left(z_{i} z_{i}^{\prime}\right)\right]^{-1} E\left(z_{i} x_{i}^{\prime}\right)\right]^{-1} .
\end{aligned}
$$

Furthermore,

$$
H_{n} \stackrel{d}{\rightarrow}(h+\xi)^{\prime} P(h+\xi)
$$

and

$$
\sqrt{n}\left(\hat{\beta}_{c}-\beta\right) \stackrel{d}{\rightarrow} G_{1}^{\prime} \xi-\left(\frac{\tau}{(h+\xi)^{\prime} P(h+\xi)}\right)_{1} G^{\prime}(h+\xi)
$$

where $P=G\left(V_{1}-V_{0}\right)^{-1} G^{\prime}, G=\left(\begin{array}{cc}-I & I\end{array}\right)^{\prime}, G_{1}=\left(\begin{array}{cc}0 & I\end{array}\right)^{\prime}$, and $(a)_{1}=\min [1, a]$.

Remark 1. Theorem 1 presents the joint asymptotic distribution of the OLS and IV estimators, the Hausman statistic, and the combined estimator under the local to zero measurement error assumption. The joint asymptotic distribution of the OLS and IV estimators is normal with a classic covariance matrix. The OLS estimator has an asymptotic bias when $\delta_{1} \neq 0$ (and thus $\delta_{2} \neq 0$ ), but the IV estimator does not have any asymptotic bias and is consistent. However, we emphasize that the theorem holds under some conditions that conventional central limit theory applies. As is well known (Staiger and Stock 1997, Bekker 1994), our Monte Carlo results show that when there are many instruments ( $\ell$ is large) or when there are weak instruments, the IV estimator is inconsistent. In that case we need to regularize the IV estimator to reduce the number of instruments and to shrink the weak instruments. Based on the regularized IV, we can then recover the theorem and construct a regularized combined estimator. We leave this for our next research agenda. 
The asymptotic risk of an estimator $\hat{\beta}$ of $\beta$ is defined as

$$
R(\hat{\beta}, \beta, W)=\lim _{n \rightarrow \infty} E\left[n(\hat{\beta}-\beta)^{\prime} W(\hat{\beta}-\beta)\right]
$$

so long as the estimator has an asymptotic distribution

$$
\sqrt{n}(\hat{\beta}-\beta) \stackrel{d}{\rightarrow} \psi
$$

for some random variable $\psi$, the asymptotic risk can be calculated using

$$
R(\hat{\beta}, \beta, W)=E\left(\psi^{\prime} W \psi\right)=\operatorname{tr}\left(W E\left(\psi \psi^{\prime}\right)\right)
$$

Define the largest eigenvalue of the matrix $W\left(V_{1}-V_{0}\right)$

$$
\lambda_{1}=\lambda_{\max }\left(W\left(V_{1}-V_{0}\right)\right)
$$

and the ratio

$$
d=\frac{\operatorname{tr}\left(W\left(V_{1}-V_{0}\right)\right)}{\lambda_{1}}
$$

Note that $1 \leq d \leq q$. In the case $W=\left(V_{1}-V_{0}\right)^{-1}, \lambda_{1}=1$ and we have the simplification $d=q$. Hansen (2017) shows that the asymptotic risk of the combined estimator is smaller than that of the IV estimator for all values of $\beta$.

Theorem 2 (Hansen 2017). Under some assumptions, if $q>2$ and $0<\tau \leq 2(q-2)$, then

$$
\begin{aligned}
& R\left(\hat{\beta}_{1}, \beta, W\right)=\operatorname{tr}\left(W V_{1}\right) \\
& R\left(\hat{\beta}_{c}, \beta, W\right)<R\left(\hat{\beta}_{1}, \beta, W\right)-\frac{\tau[2(q-2)-\tau]}{\sigma_{u}^{-4} \delta_{2}^{\prime} \Sigma_{v} V_{0}\left(V_{1}-V_{0}\right)^{-1} V_{0} \Sigma_{v} \delta_{2}+q}
\end{aligned}
$$

where $\Sigma_{v}=E\left(v_{i} v_{i}^{\prime}\right)$.

Remark 2. Theorem 2 can be stated in terms of $\delta_{1}$ using the relationship $\Sigma_{v} \delta_{2}=-\delta_{1} \beta$ in $(16)$

$$
R\left(\hat{\beta}_{c}, \beta, W\right)<R\left(\hat{\beta}_{1}, \beta, W\right)-\frac{\tau[2(q-2)-\tau]}{\sigma_{u}^{-4} \delta_{1}^{2} \beta^{\prime} V_{0}\left(V_{1}-V_{0}\right)^{-1} V_{0} \beta+q}
$$


The improvement in the asymptotic risk by the combined estimator $\hat{\beta}_{c}$ over the IV estimator $\hat{\beta}_{1}$ depends on the measurement error bias of the OLS estimator $\hat{\beta}_{0}$, as the OLS bias in (7) depends on $-\sigma_{\varepsilon_{2}}^{2} \beta=-\delta_{1} \beta / \sqrt{n}$.

\section{Monte Carlo}

We thank Bruce Hansen for his R code, which we downloaded from his website. For our measurement model we made a minor modification of his code. The observations $\left\{y_{i}, x_{i}, z_{i}\right\}_{i=1}^{n}$ are generated by the process

$$
\begin{aligned}
& y_{i}=x_{i}^{* \prime} \beta+\varepsilon_{1 i} \\
& x_{i}=x_{i}^{*}+\varepsilon_{2 i} \\
& x_{i}^{*}=\Pi z_{i}+v_{i}
\end{aligned}
$$

where $\varepsilon_{1 i} \sim N(0,1)$, the measurement errors $\varepsilon_{2 i}$ are uncorrelated each other and follows $N\left(0, \Sigma_{\varepsilon_{2}}\right), \Sigma_{\varepsilon_{2}}=\operatorname{diag}\left(\sigma_{1}^{2}, \sigma_{2}^{2}, \ldots, \sigma_{q}^{2}\right)$ is a $q \times q$ diagonal matrix of measurement error variances, $v_{i}$ is a $q \times 1$ vector following $N\left(0, I_{q}\right), z_{i}$ is an $\ell \times 1$ vector of instruments with $N\left(0, I_{\ell}\right)$ and $\ell \geq q$. We report the results only for $\ell=q$.

We set $\sigma_{1}=\sigma_{2}=\cdots=\sigma_{q}=\sigma_{\varepsilon_{2}}$ so that $\Sigma_{\varepsilon_{2}}=\sigma_{\varepsilon_{2}}^{2} I_{q}$. We have experimented with $\sigma_{\varepsilon_{2}} \in$ $[0,3]$ but report results with $\sigma_{\varepsilon_{2}}$ only on $[0,1]$. Note that our experiment sets the dimension of $z_{i}$ equal to that of $x_{i}$, so the IV estimates are just-identified $(\ell=q)$. We set the $q \times q$ reduced form matrix as $\Pi=I_{q} d$ and the scale $d$ set as $d=\sqrt{R^{2} /\left(1-R^{2}\right)}$ so that $R^{2}$ is the reduced form population $R^{2}$ for each $x_{j i}, j=1, \ldots, q$. We vary $n \in\{100,200\}, q \in\{1,2,3,5,10,20\}$, $R^{2} \in\{0.10,0.40\}$ and $\sigma_{\varepsilon_{2}}$ on a 40-point grid on [0,0.975]. The parameter $R^{2}$ controls the strength of the instruments (small $R^{2}$ is for the case of weak instruments) and the parameter $\sigma_{\varepsilon_{2}}$ controls the size of measurement errors and thus the degree of endogeneity $\left(\sigma_{\varepsilon_{2}}=0\right.$ is for the case of no measurement error and large $\sigma_{\varepsilon_{2}}$ is for the case of large measurement error). 
We generated 10,000 samples for each configuration, and on each calculated $\hat{\beta}_{0}, \hat{\beta}_{1}$ and $\hat{\beta}_{c}$. For $\hat{\beta}_{c}$, as in Hansen (2017), we set $\tau=\frac{1}{4}$ for $q=1, \tau=1$ for $q=2$, and $\tau=q-2$ for $q>2$. Our focus will be with a large $q$, such as $q=3,5,10,20$, for consideration of using many instruments. The measurement error bias in the OLS estimator $\hat{\beta}_{0}$ depends on $\sigma_{\varepsilon_{2}}^{2}$ and $\beta$. We fix $\beta=(1 \ldots 1)^{\prime}$ such that the bias is controlled only by $\sigma_{\varepsilon_{2}}^{2}$, the size of the measurement error.

To compare the estimators we calculated the weighted median squared error risk

$$
\operatorname{median}\left((\hat{\beta}-\beta)^{\prime} W(\hat{\beta}-\beta)\right)
$$

for each of the three estimators $\hat{\beta}_{0}, \hat{\beta}_{1}$ and $\hat{\beta}_{c}$. The weight is either $W=I$ or $W=$ $\left(\hat{V}_{1}-\hat{V}_{0}\right)^{-1}$. As in Hansen (2017), we report the median squared error rather than the mean squared error because IV estimators for the just-identified model $(\ell=q)$ may not have finite moments.

We produced many figures for various configurations. For space, we report only Figures $1,2,3,4$ in the paper. Figure 1 is for $n=100, W=I$. Figure 2 is for $n=100, W=$ $\left(\hat{V}_{1}-\hat{V}_{0}\right)^{-1}$. Figure 3 is for $n=200, W=I$. Figure 4 is for $n=200, W=\left(\hat{V}_{1}-\hat{V}_{0}\right)^{-1}$. For all of Figures $1,2,3,4$, we set $\sigma_{1}=\sigma_{2}=\cdots=\sigma_{q}=\sigma_{\varepsilon_{2}}$ so that $\Sigma_{\varepsilon_{2}}=\sigma_{\varepsilon_{2}}^{2} I_{q} \cdot{ }^{1}$ There are 12 subfigures in each figure. Subfigures 1 and 2 are with $q=1$. Subfigures 3 and 4 are for $q=2$. Subfigures 5 and 6 are the with $q=3$. Subfigures 7 and 8 are with $q=5$. Subfigures 9 and 10 are using $q=10$. Subfigures 11 and 12 have $q=20$. The subfigures with odd numbers are the case $R^{2}=0.1$ (weaker instruments). The subfigures with even numbers are the case $R^{2}=0.4$ (stronger instruments).

Some observations from these results are summarized here. Theorem 2 shows that the asymptotic risk of the combined estimator is uniformly smaller than that of the IV estimator

\footnotetext{
${ }^{1}$ We have also considered cases where only one (the first) regressor has measurement error, i.e., $\sigma_{1}=\sigma_{\varepsilon_{2}}$ and $\sigma_{2}=\cdots=\sigma_{q}=0$ so that $\Sigma_{\varepsilon_{2}}=\operatorname{diag}\left(\sigma_{\varepsilon_{2}}^{2}, \mathbf{0}\right)$. These are Figures $5,6,7,8$, in the supplemental appendix, available in our website.
} 
when $q>2$. Subfigures for $q>2$ show that the asymptotic uniform ranking holds in finite samples. When $q$ is too large $(q=10,20)$, subfigures $1.9,1.10,1.11$ and 1.12 show that both OLS and IV are inconsistent, little or no improvements can be achieved by the combined estimator as the combined estimator puts (almost) all weight on OLS and little weight on IV as $q$ gets larger relative to $n$. It is interesting that IV is worse than OLS when $q$ is large, for a quite large size of measurement errors over a wide range of $\sigma_{\varepsilon_{2}}$. It should be note that, when IV loses consistency due to many instruments, the Hausman statistic may not be used for the construction of the combined estimator.

Finally, in order to see if this would also happen with other form of endogeneity, we also replicated Hansen's (2017) monte carlo results for his setup of a structural model using large $q=5,10,20 .^{2}$ In Hansen (2017), it is reported only for small values of $q \in\{1,2,3,4\}$. As expected, for larger values of $q$, the findings from the structural model are similar to what we see from Figures 1, 2, 3, 4 for the measurement error model: namely, when $q$ is large, both OLS and IV are inconsistent, IV is worse than OLS, and little or no improvements can be achieved by the combined estimator.

\section{Conclusions}

Given the findings from this paper, a next question is when we may use IV. When does IV do better than OLS? We find that's when $R^{2}$ is not small (when instruments are not weak), $\sigma_{\varepsilon_{2}}^{2}$ is not small (when measurement errors are large), and $q$ is not large relative to $n$ (when there are not too many instruments). Otherwise, IV can be bad, possibly much worse than OLS. When we do not know how small $R^{2}$ or $\sigma_{\varepsilon_{2}}^{2}$ is too small or how large $q$ is too large, a safe guard would be to use the combined estimator. Although these findings are shown for the errors in variables model they may apply to other econometric models using IV estimators.

Recently, many papers have been written to deal with the endogeneity in high dimen-

\footnotetext{
${ }^{2}$ These are Figures 9 and 10 in the supplemental appendix, available in our website.
} 
sional models with large $q$. The basic idea is to reduce the dimension $q$ by selecting some of the instruments. The lasso type methods pioneered by Tibshirani (1996) reduce the number of variables in the high dimensional models by shrinking some of the estimators toward zero. With the rapid advance of statistical learning and machine learning in big data environment, the regularization of the standard econometric methods such as OLS, IV or GMM have been very actively studied in recent econometrics community, for example, Friedman (2001), Bühlmann and Yu (2003), Chao and Swanson (2007), Hansen, Donald, Imbens, and Newey (2008), Hausman, and Newey (2008), Hansen, Hausman and Newey (2008), Ng and Bai (2008), Newey and Windmeijer (2009), Caner (2009), Donald, Imbens, and Newey (2009), Carrasco (2012), Belloni, Chen, Chernozhukov, and Hansen (2012), Belloni and Chernozhukov (2011, 2013), Liao (2013), Fan and Liao (2014), Chernozhukov and Hansen (2004, 2005, 2006, 2013), Chernozhukov, Hansen, and Spindler (2015), Cheng and Liao (2015), DiTraglia (2016), Harding, Hausman, and Palmer (2016), Chudik, Kapetanios and Pesaran (2016), Caner, Han, and Lee (2017), Caner, Maasoumi, and Riquelme (2017), and Lee and Xu (2017), among others. They show various methods such as lasso, adaptive lasso, SCAD, elastic net, boosting, to mitigate the effect of high dimension on consistent estimation of the econometric models such as the conditional mean and conditional quantiles. Many of these papers have studied various regularization methods to select instruments to improve IV. It would be interesting to see how these methods for high dimensional models could also improve the combined estimator. As noted in Remark 1 above, we are currently investigating this in relation to the current paper and Hansen (2017). 


\section{References}

Bekker, P.A. (1994). "Alternative approximations to the distributions of instrumental variable estimators", Econometrica 62, 657-681.

Belloni, A., Chen, D., Chernozhukov, V., and Hansen, C. (2012). "Sparse Models and Methods for Optimal Instruments with an Application to Eminent Domain", Econometrica $80,2369-2429$.

Belloni, A. and Chernozhukov, V. (2011). " $\ell_{1}$-penalized quantile regression in high-dimensional sparse models," Annals of Statististics 39(1), 82-130.

Belloni, A. and Chernozhukov, V. (2013). "Least Squares After Model Selection in Highdimensional Sparse Models", Bernoulli 19(2), 521-547.

Bühlmann, P. and Yu, B. (2003). "Boosting with the $L_{2}$ Loss". Journal of the American Statistical Association 98, 324-339.

Caner, M. (2009), "Lasso-type GMM Estimator". Econometric Theory 25(1): 270-290

Caner, M., Han, X., and Lee, Y. (2017). "Adaptive Elastic Net GMM Estimation with Many Invalid Moment Conditions: Simultaneous Model and Moment Selection". Journal of Business and Economic Statistics, forthcoming.

Caner, M., Maasoumi, E., and Riquelme, J. (2017). "Moment and IV Selection Approaches: A Comparative Simulation Study". Econometric Reviews, forthcoming.

Carrasco, M. (2012). "A Regularization Approach to the Many Instruments Problem". Journal of Econometrics 170, 383-398

Chao, J. and Swanson, N. (2007). "Alternative specifications of the bias and MSE of the IV estimator under weak identification with an application to bias correction." Journal of Econometrics 137, 515-555.

Chernozhukov, V. and Hansen, C. (2004), "The effects of 401(k) participation on the wealth distribution: An instrumental quantile regression analysis." The Review of Economics and Statistics 86(3), $735-751$.

Chernozhukov, V. and Hansen, C. (2005). "An IV model of quantile treatment effects". Econometrica 73 (1), 245- 261. 
Chernozhukov, V. and Hansen, C. (2006). "Instrumental quantile regression inference for structural and treatment effects models ". Journal of Econometrics 132, 491 -525.

Chernozhukov, V. and Hansen, C. (2013). "Quantile models with endogeneity". Annual Review of Economics 5(1), 57 -81.

Chernozhukov, V., Hansen, C., and Spindler, M. (2015). "Post-Selection and Post-Regularization Inference in Linear Models with Many Controls and Instruments". MIT Department of Economics Working Paper No. 15-02.

Cheng, X. and Liao, Z. (2015). "Select the Valid and Relevant Moments: An Informationbased LASSO for GMM with Many Moments". Journal of Econometrics 186, 443-464

Chudik, A., Kapetanios, G. and Pesaran, M.H. (2016), "Big Data Analytics: A New Perspective." USC Globalization and Monetary Policy Institute Working Paper 268.

DiTraglia, F. (2016). "Using Invalid Instruments on Purpose: Focused Moment Selection and Averaging for GMM." Journal of Econometrics 195(2), 187-208.

Donald., S., Imbens, G., and Newey, W. (2008). "Choosing Instrumental Variables in Conditional Moment Restriction Models". Journal of Econometrics. 152, 28-36.

Fan, J. and Runze Li (2001), "Variable Selection via Nonconcave Penalized Likelihood and its Oracle Properties ". Journal of the American Statistical Association, 96(456): $1348-1360$

Fan, J. and Liao, Y. (2014). "Endogeneity in high dimension." The Annals of Statistics $42(3), 872-917$.

Friedman, J. (2001), "Greedy Function Approximation: A Gradient Boosting Machine." The Annals of Statistics 29(5): 1189-1232.

Hansen, B.E. (2017). "A Stein-Like 2SLS Estimator." Econometric Reviews forthcoming. http://www.ssc.wisc.edu/ bhansen/papers/shrinkiv.pdf

Hansen, C., Hausman, J., and Newey, W. (2008), "Estimation with many instrumental variables." Journal of Business $\&$ Economic Statistics 26(4), 398 - 422.

Harding, M., Hausman, J. and Palmer, C.J. (2016), "Finite sample bias corrected IV estimation for weak and many instruments". Advances in Econometrics 36, 245 - 273. 
Hausman, J.A. (1978). "Specification tests in econometrics." Econometrica 46(6), 12511271.

James, W. and Stein, C.M. (1961). "Estimation with quadratic loss." Proceedings of the Fourth Berkeley Symposium on Mathematical Statistics and Probability, Vol. 1, 361379.

Lee, T-H and Xu, H. (2017). "Boosting Penalized GMM Estimation with Many Instruments That Are Possibly Irrelevant Or Invalid". UC Riverside.

Newey, W.K. and Windmeijer, F. (2009). "Generalized method of moments with many weak moment conditions". Econometrica 77(3), 687-719.

Ng, S. and Bai, J. (2008). "Selecting Instrumental Variables in a Data Rich Environment". Journal of Time Series Econometrics 1(1), Article 4.

Staiger, D. and Stock, J (1997). "Instrumental Variables Regression with Weak Instruments ". Econometrica 65(3), 557-586.

Tibshirani, R. (1996). "Regression Shrinkage and Selection via the Lasso". Journal of Royal Statististical Society, Series B 58, 267-288. 


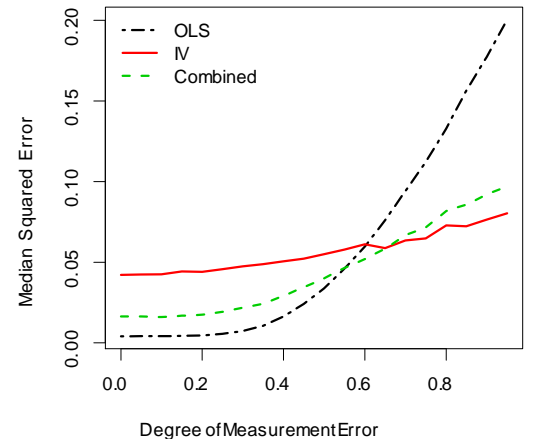

$1.1: q=1, R^{2}=0.1$

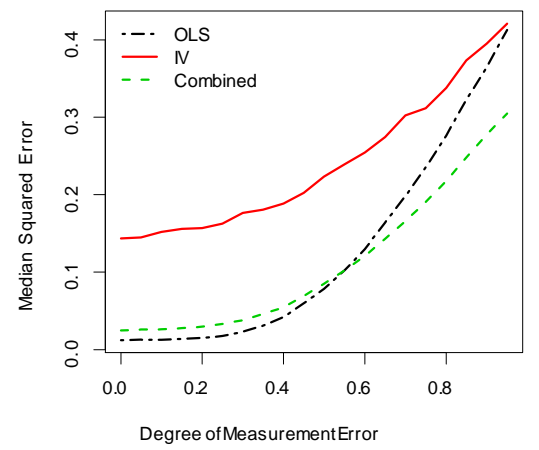

$1.3: q=2, R^{2}=0.1$

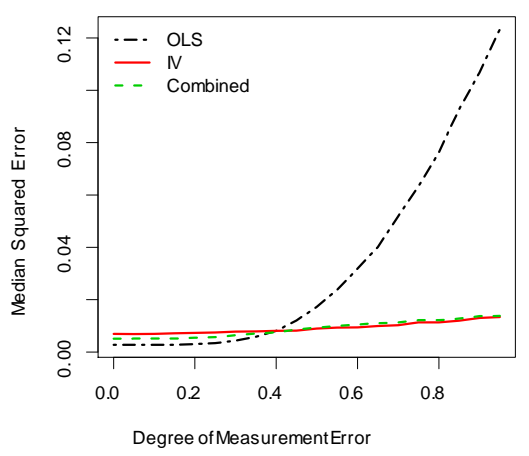

$1.2: q=1, R^{2}=0.4$

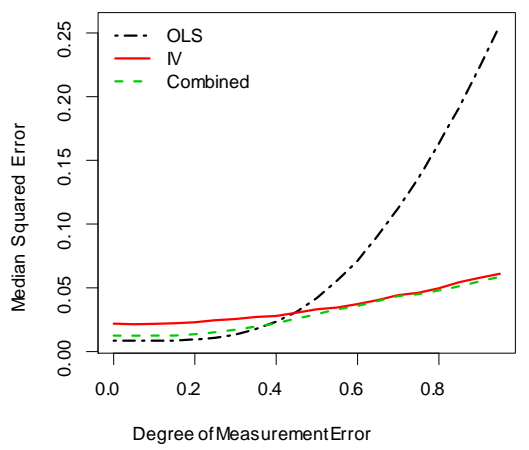

$1.4: q=2, R^{2}=0.4$

Figure 1: $n=100, W=I$ 


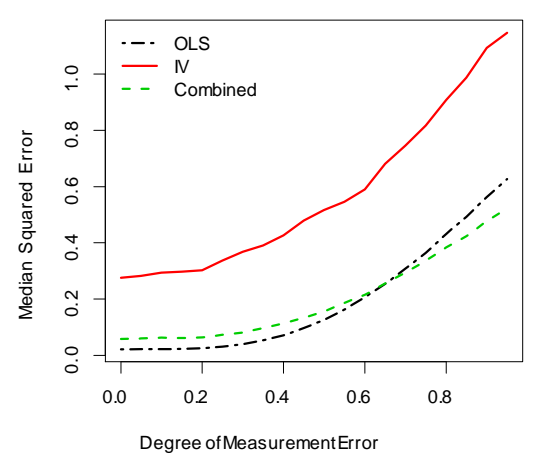

$1.5: q=3, R^{2}=0.1$

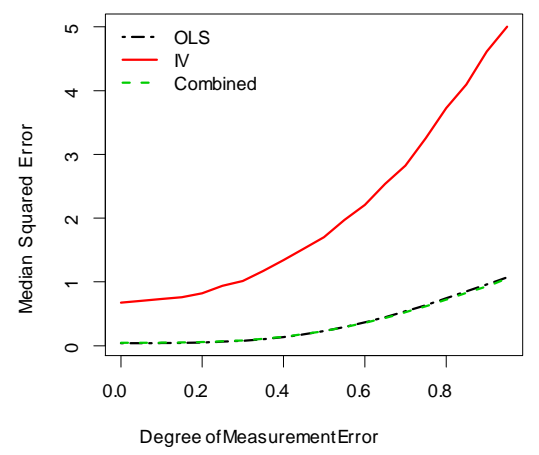

$1.7: q=5, R^{2}=0.1$

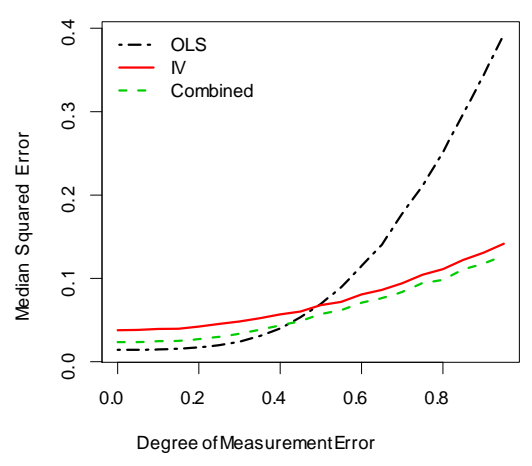

$1.6: q=3, R^{2}=0.4$

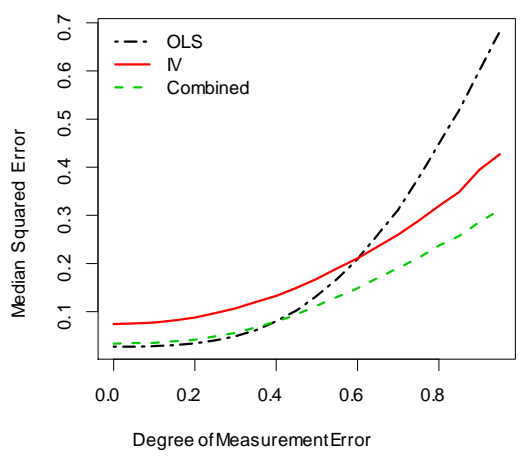

$1.8: q=5, R^{2}=0.4$

Figure 1: $n=100, W=I$ 


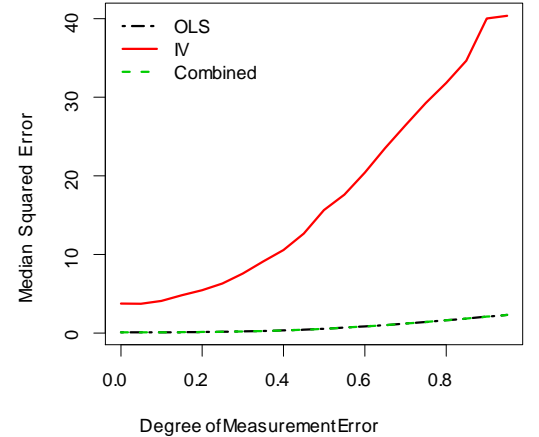

$1.9: q=10, R^{2}=0.1$

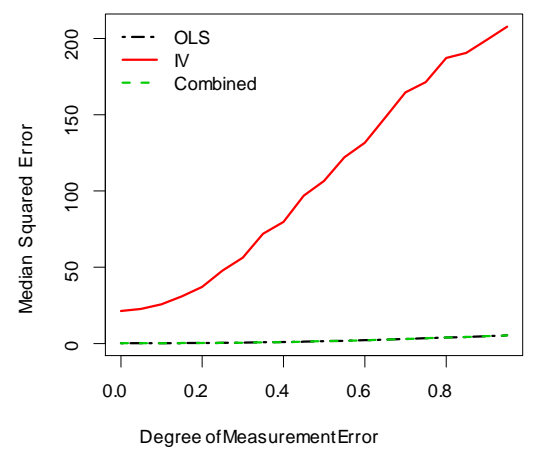

$1.11: q=20, R^{2}=0.1$

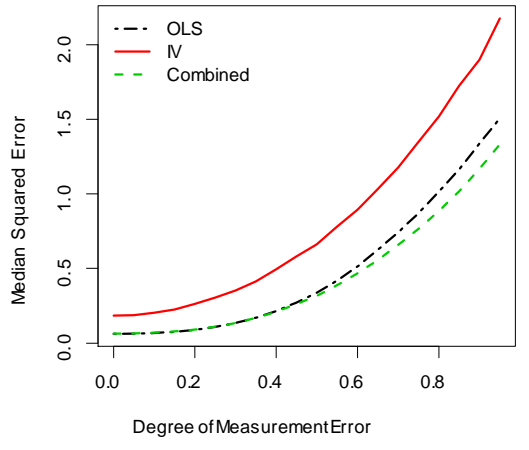

$1.10: q=10, R^{2}=0.4$

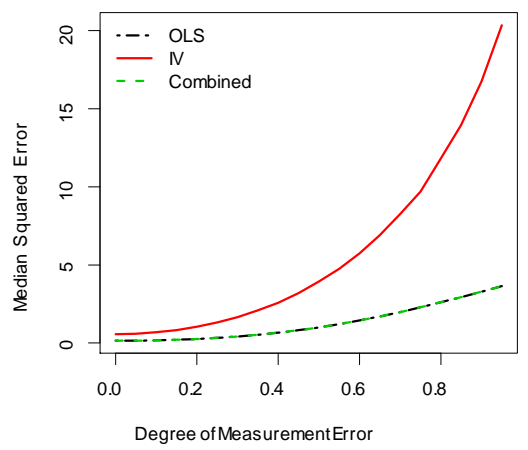

$1.12: q=20, R^{2}=0.4$

Figure 1: $n=100, W=I$ 


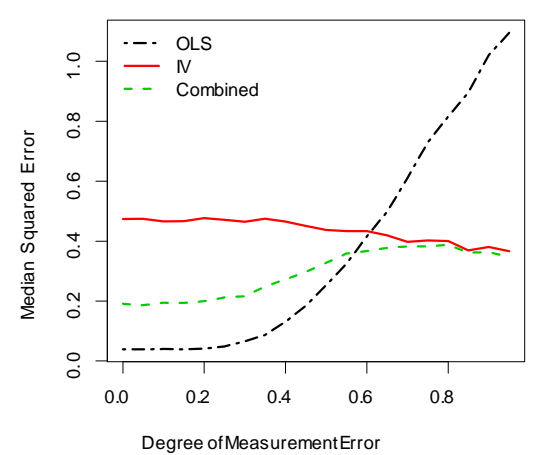

$2.1: q=1, R^{2}=0.1$

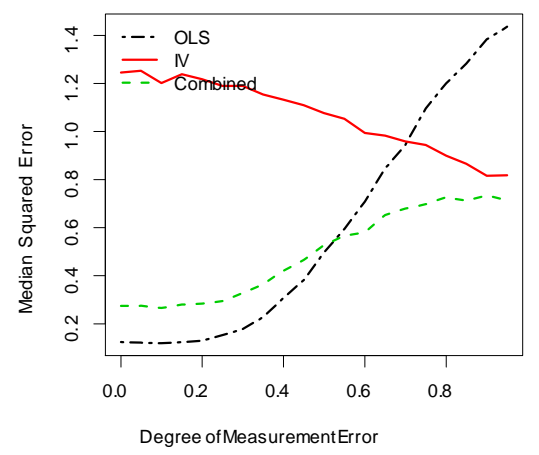

$2.3: q=2, R^{2}=0.1$

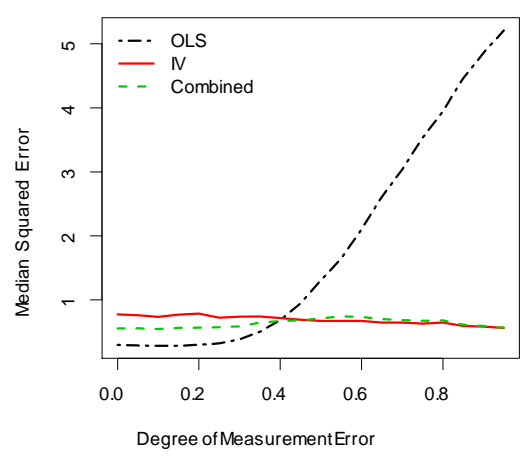

$2.2: q=1, R^{2}=0.4$

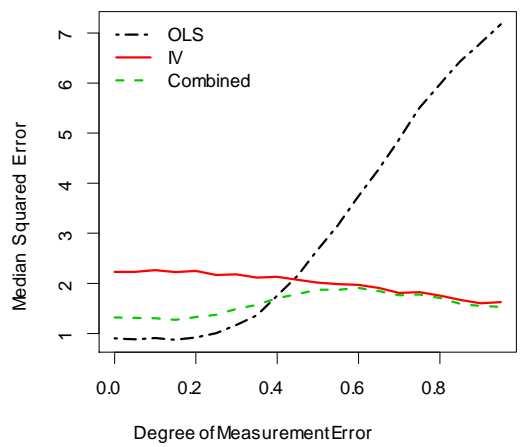

$2.4: q=2, R^{2}=0.4$

Figure $2: n=100, W=\left(\hat{V}_{1}-\hat{V}_{0}\right)^{-1}$ 


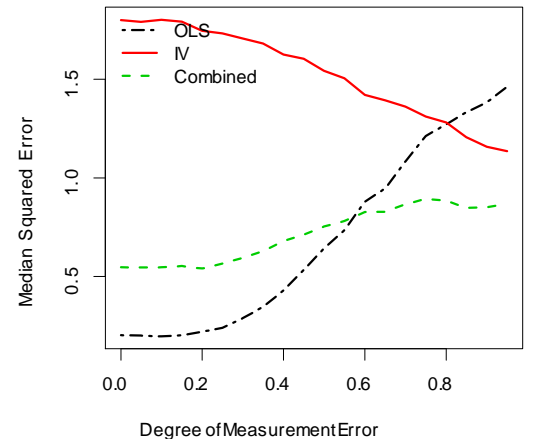

$2.5: q=3, R^{2}=0.1$

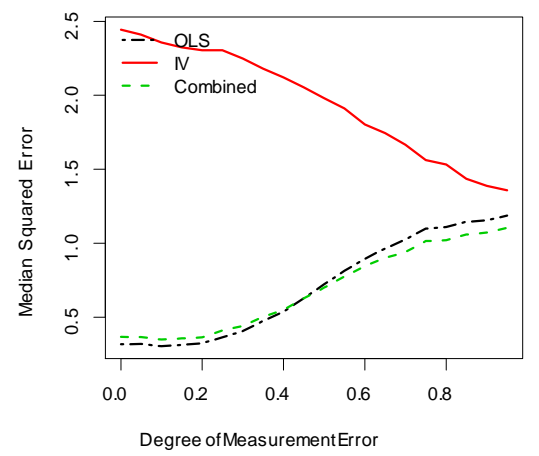

$2.7: q=5, R^{2}=0.1$

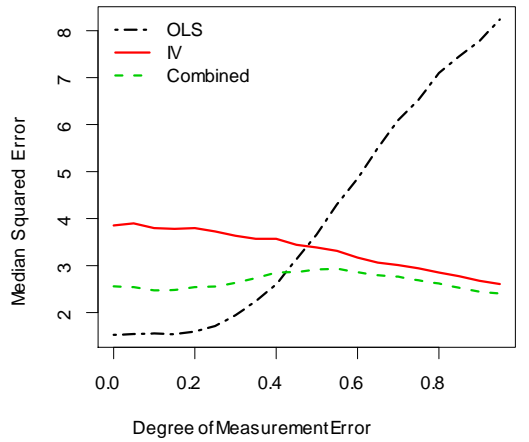

$2.6: q=3, R^{2}=0.4$

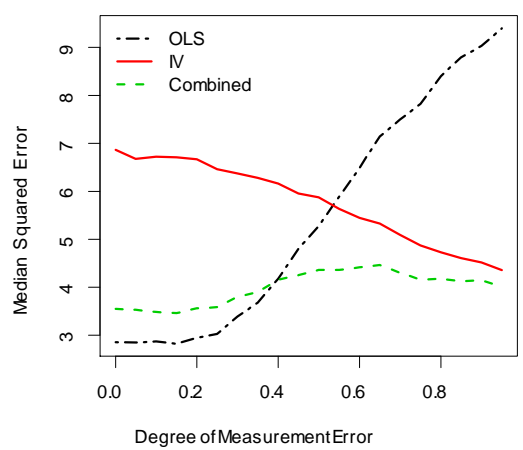

$2.8: q=5, R^{2}=0.4$

Figure 2: $n=100, W=\left(\hat{V}_{1}-\hat{V}_{0}\right)^{-1}$ 


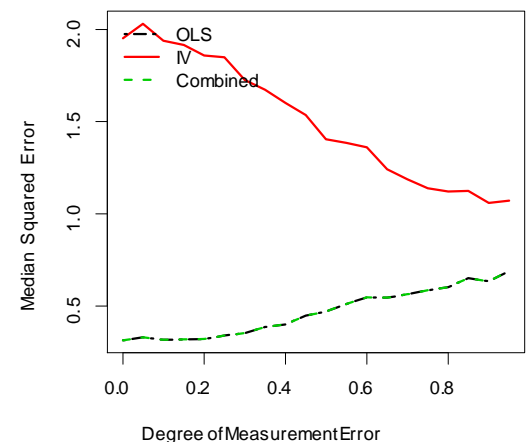

$2.9: q=10, R^{2}=0.1$

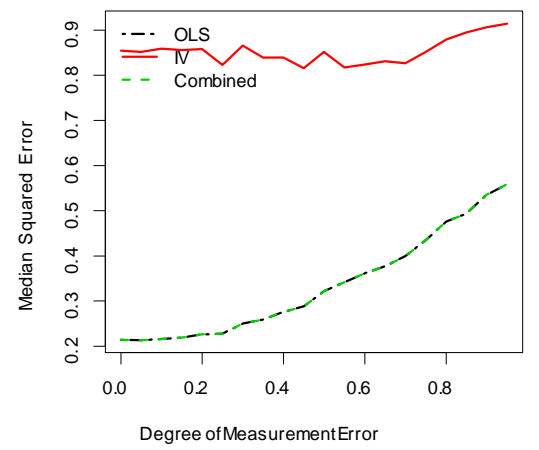

$2.11: q=20, R^{2}=0.1$

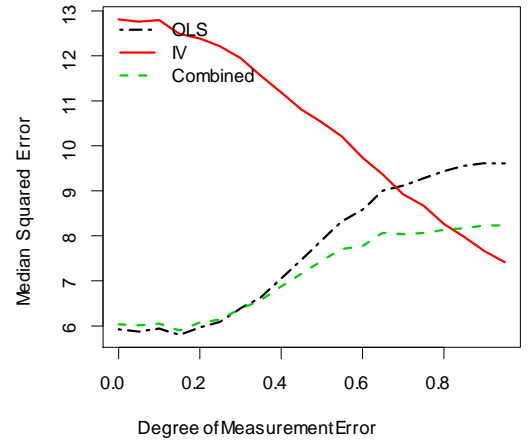

$2.10: q=10, R^{2}=0.4$

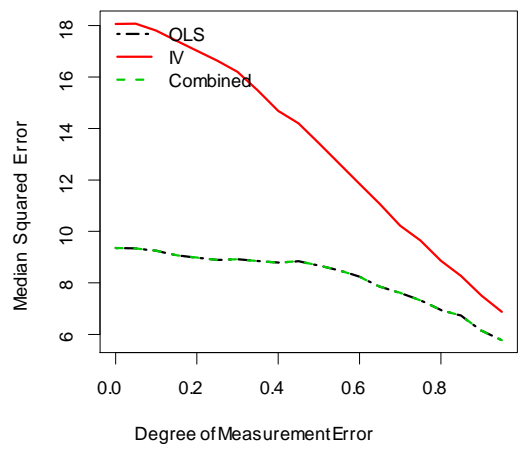

$2.12: q=20, R^{2}=0.4$

Figure 2: $n=100, W=\left(\hat{V}_{1}-\hat{V}_{0}\right)^{-1}$ 


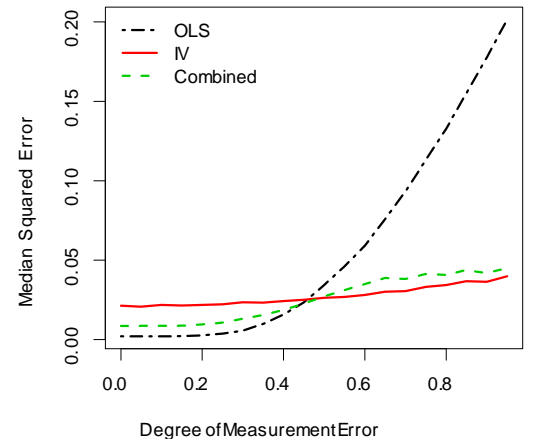

$3.1: q=1, R^{2}=0.1$

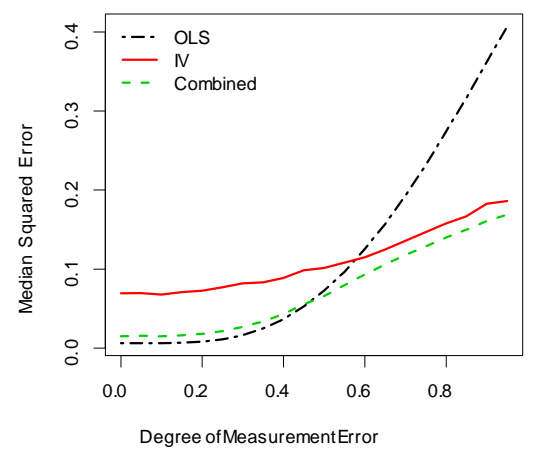

$3.3: q=2, R^{2}=0.1$

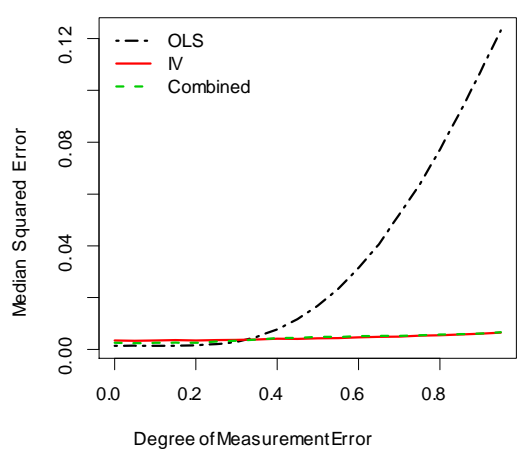

$3.2: q=1, R^{2}=0.4$

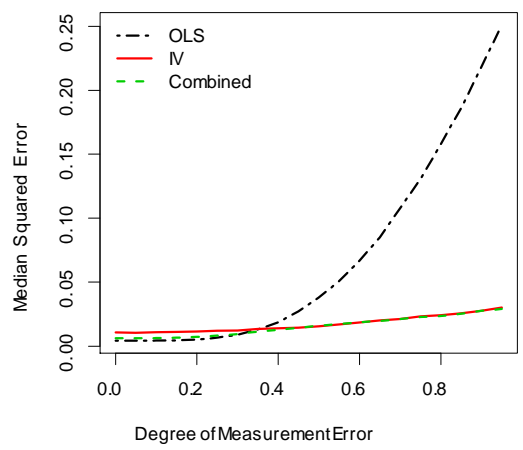

$3.4: q=2, R^{2}=0.4$

Figure 3: $n=200, W=I$ 


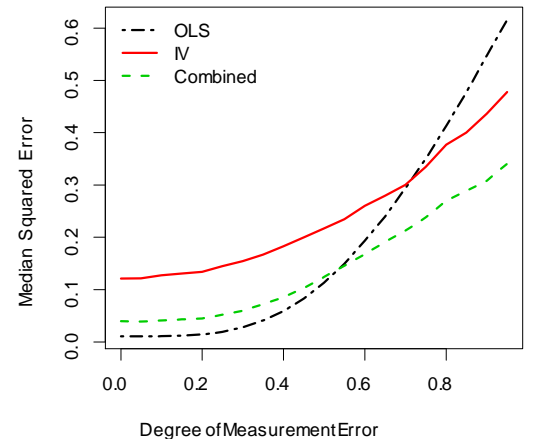

$3.5: q=3, R^{2}=0.1$

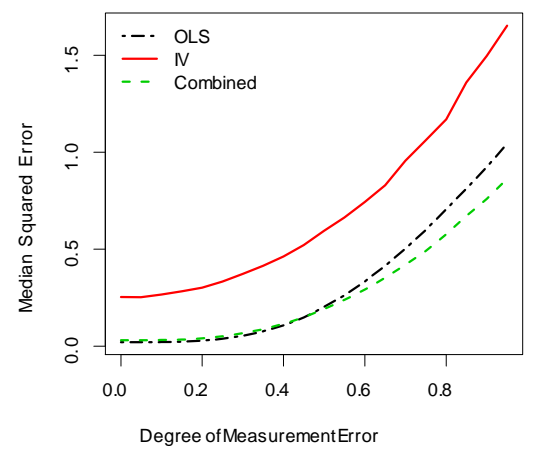

$3.7: q=5, R^{2}=0.1$

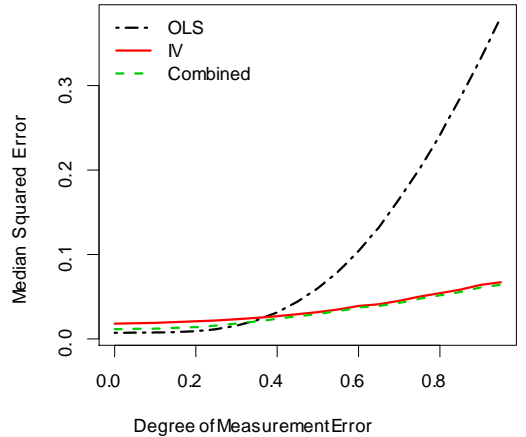

$3.6: q=3, R^{2}=0.4$

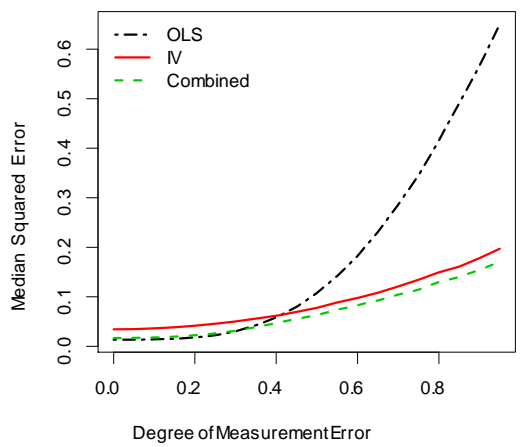

$3.8: q=5, R^{2}=0.4$

Figure 3: $n=200, W=I$ 


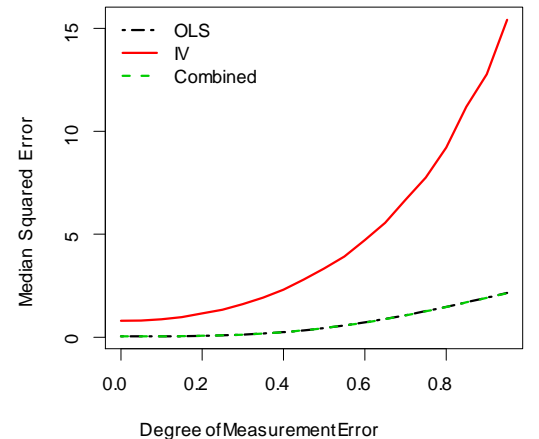

$3.9: q=10, R^{2}=0.1$

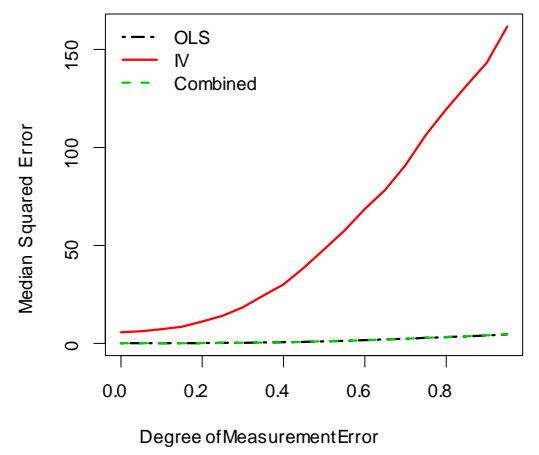

$3.11: q=20, R^{2}=0.1$

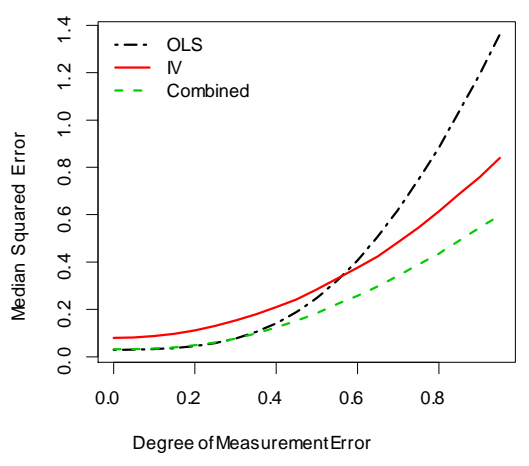

$3.10: q=10, R^{2}=0.4$

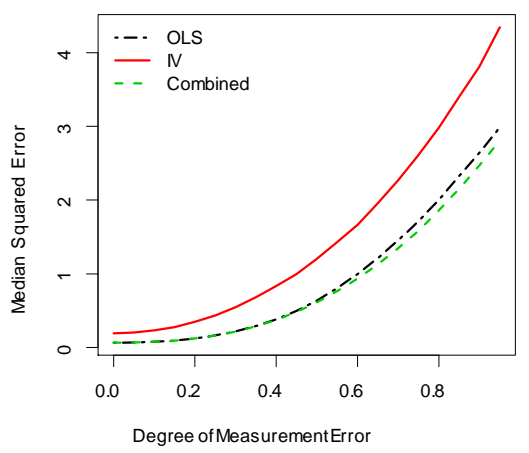

$3.12: q=20, R^{2}=0.4$

Figure 3: $n=200, W=I$ 


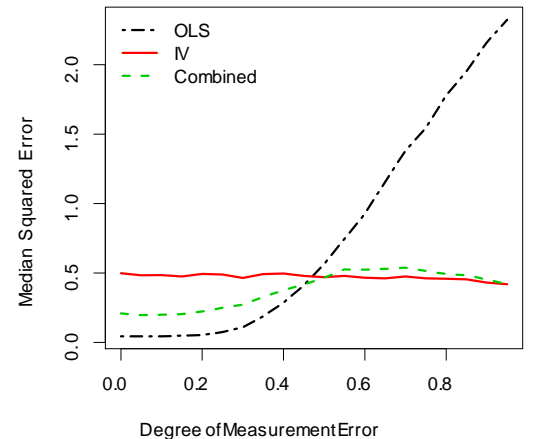

$4.1: q=1, R^{2}=0.1$

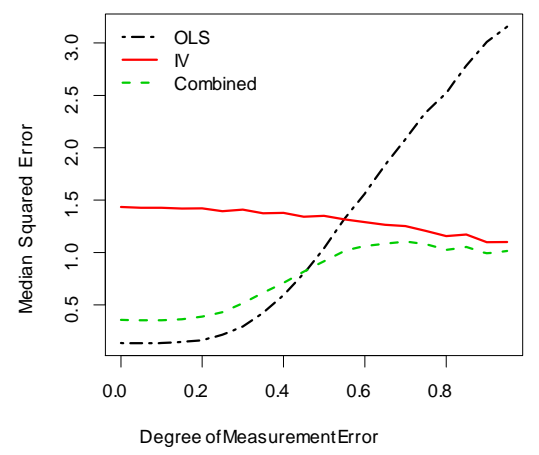

$4.3: q=2, R^{2}=0.1$

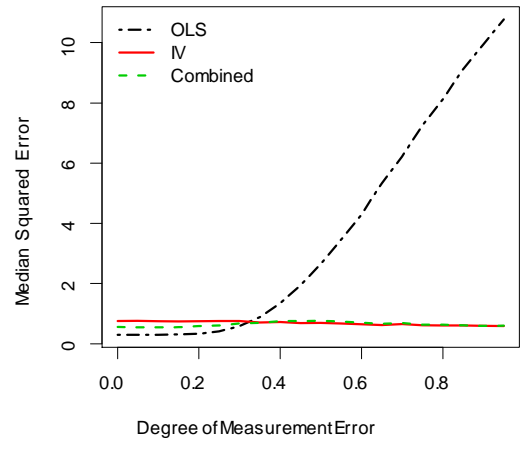

$4.2: q=1, R^{2}=0.4$

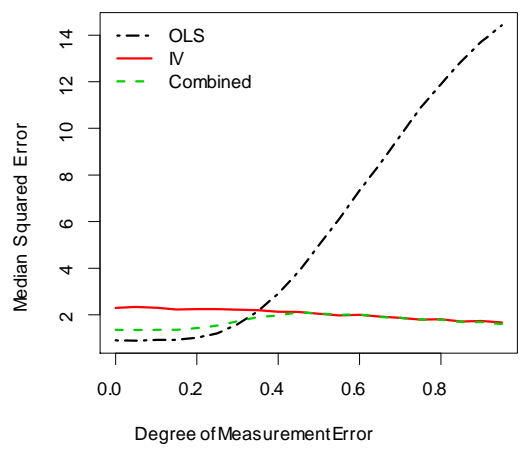

$4.4: q=2, R^{2}=0.1$

Figure 4: $n=200, W=\left(\hat{V}_{1}-\hat{V}_{0}\right)^{-1}$ 


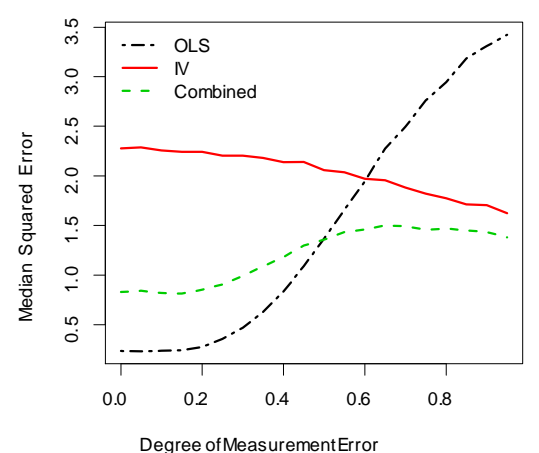

$4.5: q=3, R^{2}=0.1$

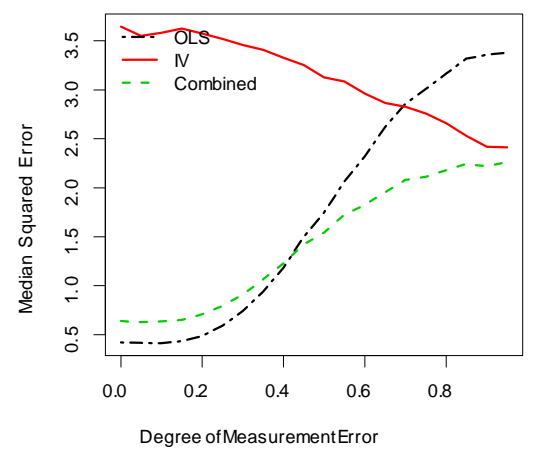

$4.7: q=5, R^{2}=0.1$

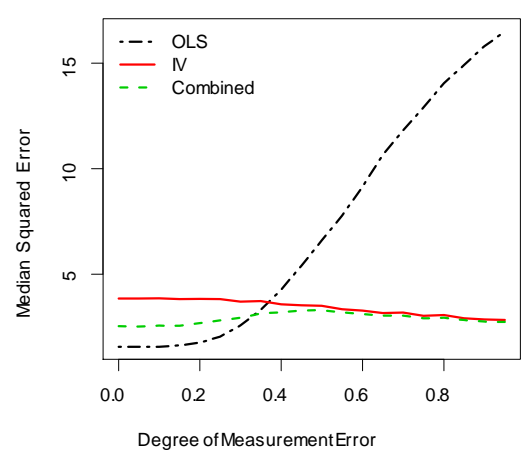

$4.6: q=3, R^{2}=0.4$

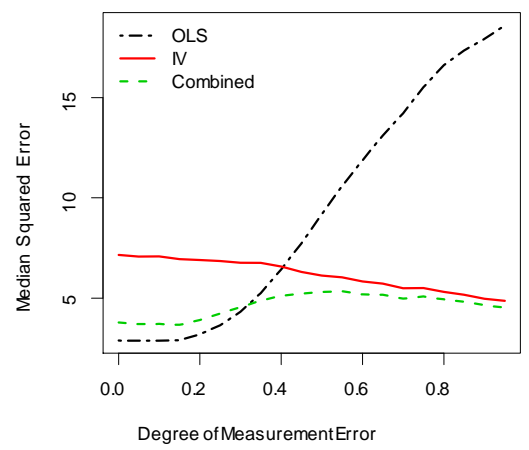

$4.8: q=5, R^{2}=0.4$

Figure 4: $n=200, W=\left(\hat{V}_{1}-\hat{V}_{0}\right)^{-1}$ 


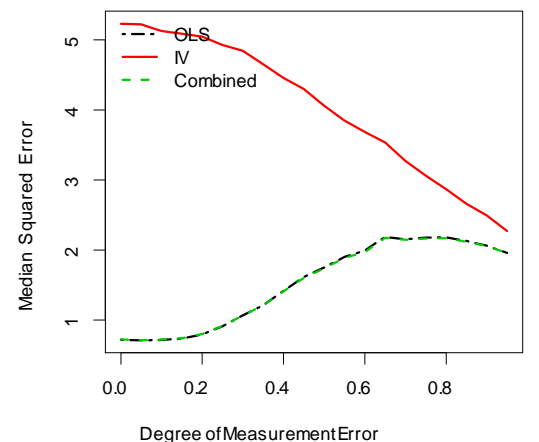

$4.9: q=10, R^{2}=0.1$

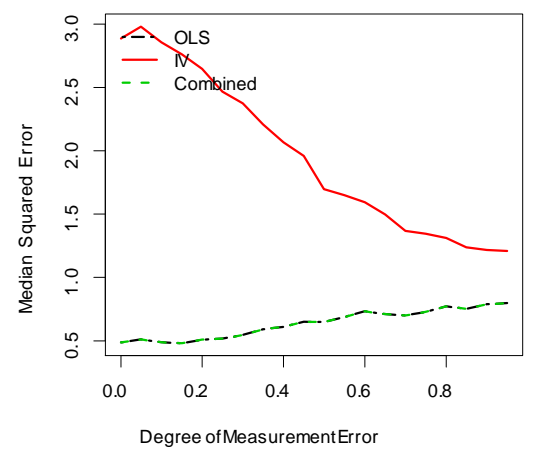

$4.10: q=20, R^{2}=0.1$

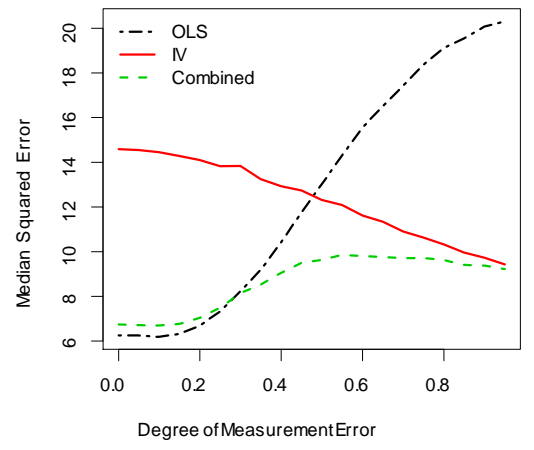

$4.10: q=10, R^{2}=0.4$

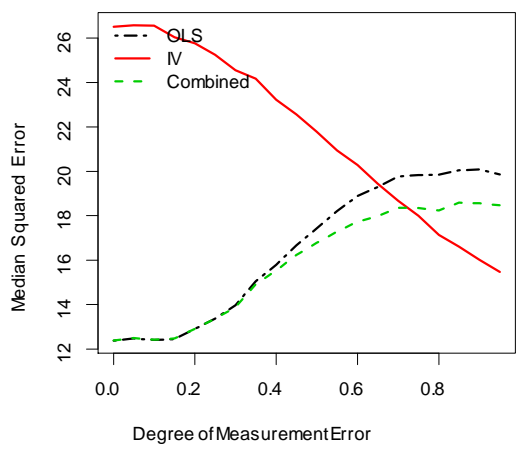

$4.12: q=20, R^{2}=0.4$

Figure 4: $n=200, W=\left(\hat{V}_{1}-\hat{V}_{0}\right)^{-1}$ 